Series A

\author{
I. MATHEMATICA
}

$336 / 13$

\title{
VERALLGEMEINERTE POISSON_STIELTJES'SCHE INTEGRALDARSTELLUNG UND KONTRAKTIVE OPERATOREN
}

voN

ALBERT PFLUGER

H E L S I N I 1963

S U O M A L A I EN T I E D A K A T EM I A 
Am 13. September 1963 vorgelegt von P. J. Myrberg und Olli Lehto 


\section{Verallgemeinerte Poisson-Stieltjes'sche Integraldarstellung und kontraktive Operatoren}

Die Spektraldarstellung unitärer Operatoren $\quad U$ in einem Hilbertraum $\mathfrak{H}$ kann dadurch gewonnen werden, dass man für $x \in \mathfrak{\mathfrak { Z }}$ die komplexwertige Funktion

$$
f(\lambda)=\left((E+\lambda U)(E-\lambda U)^{-1} x, x\right)
$$

betrachtet. Sie ist im Einheitskreis $\{\lambda|| \lambda \mid<1\}$ holomorph und hat dort positiven Realteil. Vermittels der Poisson-Stieltjes'schen Integraldarstellung solcher Funktionen für jedes $x \in \mathfrak{H}$ folgt dann die Existenz einer Spektralschar $\Phi$ auf dem Intervall $[0,2 \pi]$, sodass

$$
(E+\lambda U)(E-\lambda U)^{-1}=\int_{0}^{2 \pi} \frac{e^{i \vartheta}+\lambda}{e^{i \vartheta}-\lambda} d \Phi(\vartheta)
$$

ist für alle $\lambda$ mit $|\lambda| \neq 1$. Diese Darstellung einer holomorphen Funktion mit Werten in einer Banach'schen Algebra legt es nahe zu fragen, unter welchen allgemeinen Voraussetzungen (d. h. unabhängig vom speziellen Hintergrund der unitären Operatoren) eine solche Darstellung gültig sei. Es zeigt sich, dass

1. dies unter sehr allgemeinen Verhältnissen möglich ist, dass insbesondere die obige Gleichung und daraus die Spektraldarstellung unitärer Operatoren sich direkt, d. h. ohne Rückgriff auf den Hilbertraum und die komplex-analytischen Funktionen, gewinnen lassen, und dass

2. für kontraktive Operatoren und speziell für isometrische Operatoren eine analoge Darstellung mit Hilfe einer verallgemeinerten Zerlegung der Einheit gilt. Dies liefert dann auch eine verallgemeinerte Spektraldarstellung maximaler symmetrischer Operatoren ohne über den gegebenen Hilbertraum hinaus gehen zu müssen (vgl. [2], [3]).

1. Der Raum $\Re$. Unter $\Re$ verstehen wir eine Menge (ihre Elemente sind mit $A, B, \ldots, Z$ bezeichnet) zusammen mit den darin nachfolgend postulierten Strukturen und Verträglichkeitsbedingungen:

1. $\Re$ ist ein Banach'scher Raum über dem reellen Zahlkörper $\boldsymbol{R}$. $O$ bezeichnet den Nullvektor. 
2. $\Re$ ist teilweise geordnet. Die Elemente $\geqq O$ heissen positiv. Diese Ordnung ist vollständig in folgendem Sinn: jede fallende Folge von positiven Elementen besitzt eine untere Grenze.

3. Die Ordnung ist mit der algebraischen Struktur von $\Re$ verträglich: aus $A \geqq B$ folgt $A+C \geqq B+C$ für jedes $C \in \Re$ und $\lambda A \geqq \lambda B$ für jedes positive $\lambda \in R$.

4. Die Ordnung ist verträglich mit der metrischen Struktur in $\mathfrak{R}$ :

a) aus $-A \leqq B \leqq A$ folgt $\|B\| \leqq\|A\|$ und

b) aus $\lim _{n \rightarrow \infty}\left\|A_{n}-A\right\|=0$ und $A_{n} \geqq B$ folgt $A \geqq B$.

Bemerkung. In bezug auf die Zuordnung: fallende Folge $\rightarrow$ untere Grenze gilt: Sind $\left(A_{n}\right)$ und $\left(B_{n}\right)$ fallende und nach unter beschränkte Folgen, so ist $\inf \left(A_{n}\right)+\inf \left(B_{n}\right)=\inf \left(A_{n}+B_{n}\right)$ und $\lambda \inf \left(A_{n}\right)=\inf \left(\lambda_{A_{n}}\right)$ für jedes positive $\lambda \in R$.

Beispiele:

1. Der Raum $C(X)$ der reellwertigen stetigen und beschränkten Funktionen $f$ auf einem topologischen Raum $X$ mit

$$
\|f\|=\sup _{x \in X}|f(x)|
$$

und $f \geqq g$ im Sinne von $f(x) \geqq g(x)$ für alle $x \in X$.

2. Die reellen Räume $L_{p}(a, b), \quad 1 \leqq p \leqq \infty$, mit der Ordnungsrelation $f \geqq g$ im Sinne von $f(x) \geqq g(x)$ für fast alle $x \in[a, b]$.

3. Die Hermite'schen Operatoren auf einem Hilbertraum $\mathfrak{S}:$ in bezug auf die Addition solcher Operatoren, deren Multiplikation mit reellen Zahlen und deren üblichen Norm bilden sie einen reellen Banach'schen Raum; er ist teilweise geordnet durch die Relation $A \geqq B$ im Sinne von $(A x, x) \geqq(B x, x)$ für alle $x \in \mathfrak{S} ;$ die obigen Bedingungen $2,3,4$ sind erfüllt.

2. Ein Lemma. Es gilt das folgende

Lemma. Es sei $\Lambda$ eine positive lineare Abbildung des Raumes $C[a, b]$ (der reellwertigen stetigen Funktionen auf dem Intervall $[a, b])$ in den Raum $\mathfrak{R}, \quad$ d.h. $\Lambda$ sei linear und $\Lambda(f) \geqq O$ für $f \geqq 0$. Dann gibt es eine wachsende Abbildung $\Phi$ von $[a, b]$ in $\Re$, sodass für alle $f \in C[a, b]$ gilt

$$
\Lambda(f)=\int_{a}^{b} f(x) d \Phi(x) .
$$

Bemerkung 1. Das Integral ist wie im Fall eines reellwertigen $\Phi \mathbf{z u}$ verstehen: Es sei $\&$ : $a=x_{0}<x_{1}<\ldots<x_{n}=b$ eine Einteilung von $[a, b],|\mathfrak{\xi}|=\max _{0<k \leqq n}\left(x_{k}-x_{k-1}\right)$ und $\xi=\left(\xi_{1}, \ldots, \xi_{n}\right), \xi_{k} \in\left[x_{k-1}, x_{k}\right]$. Dann gibt es eine positive Funktion $\delta$ auf $(0, b-a)$ sowie ein $A$ in 
$\Re$, und dieses $A$ ist definitionsgemäss das obige Integral (1), sodass für $|\mathfrak{E}|<\delta(\varepsilon)$ die Summen

$$
S(\mathcal{E}, \xi)=\sum_{k=1}^{n} f\left(\xi_{k}\right)\left(\Phi\left(x_{k}\right)-\Phi\left(x_{k-1}\right)\right)
$$

in einer Kugel vom Radius $\varepsilon$ und Mittelpunkt $A$ liegen. Dies beruht auf folgendem: zu beliebigen Einteilungen $\mathbb{F}$ und $\mathfrak{F}^{\prime}$, mit $|\mathfrak{F}|$ und $\left|\mathfrak{E}^{\prime}\right|$ genügend klein, gibt es eine Treppenfuktion $t$, deren Sprungstellen nur in den Teilpunkten von $\&$ und $\mathfrak{F}^{\prime}$ auftreten und für die $t<f<t+\varepsilon$ ist. Gemäss Nr. 1 folgt daraus

$$
-\varepsilon(\Phi(b)-\Phi(a)) \leqq S\left(\mathbb{E}^{\prime}, \xi^{\prime}\right)-S(\mathfrak{E}, \xi) \leqq \varepsilon(\Phi(b)-\Phi(a))
$$

und nach Bedingung $4 \mathrm{a}$ in Nr. 1

$$
\left\|S\left(\mathbb{F}^{\prime}, \xi^{\prime}\right)-S(\mathfrak{E}, \xi)\right\| \leqq \varepsilon\|\Phi(b)-\Phi(a)\| .
$$

Somit konvergiert der Durchmesser der Menge

$$
M(\delta)=\{S(\mathbb{E}, \xi)|| \mathbb{E} \mid<\delta\}
$$

mit $\delta$ gegen Null und wegen $M(\delta) \subset M\left(\delta^{\prime}\right)$ für $\delta<\delta^{\prime}$ gibt es genau einen Punkt $A \in \Re$, der in allen $\overline{M(\delta)}$ enthalten ist.

Bemerkung 2. Die Menge der reellen Zahlen mit den üblichen Strukturen ist ein spezieller Raum $\mathfrak{R}$. Für diesen ist das Lemma ein bekannter Satz von F. Riesz.

Bemerkung 3. Das Lemma ist auch richtig, wenn $C[a, b]$ den Raum der komplexwertigen stetigen Funktionen auf $[a, b]$ bezeichnet. Denn die Beschränkung von $\Lambda$ auf die reellen Funktionen ergibt ein $\Phi$ mit (1) für reelles $f$. Ist $f=g+i h, g$ und $h$ reell, und setzt man

$$
\int f d \Phi=\int g d \Phi+i \int h d \Phi,
$$

so folgt aus der Linearität von $\Lambda$ die Gleichung (1) auch für komplexe $f$. Ferner gilt die Ungleichung

$$
\|\Lambda(f)\|<2\|\Lambda(1)\|\|f\|, \quad\|f\|=\max _{[a, b]}|f(x)| .
$$

Denn es ist

$$
\begin{aligned}
\|\Lambda(f)\| & \leqq\|\Lambda(g)\|+\|\Lambda(h)\| \\
& \leqq\|\Lambda(1)\|(\|g\|+\|h\|)<2\|\Lambda(1)\|\|f\| .
\end{aligned}
$$

3. Beweis des Lemmas. 1) Konstruktion von $\Phi$. Wir setzen $\Phi(a)=O . \quad \varphi(. ; x) \quad$ sei die charakteristische Funktion des Intervalls 
$[a, x], x \in(a, b]$. Die Funktionen $f_{n}(. ; x), n=1,2, \ldots, x \in(a, b]$ sind definiert durch Beschränkung der nachfolgenden Festsetzungen auf das Intervall $a \leqq \xi \leqq b$ :

$$
f_{n}(\xi ; x)=\left\{\begin{array}{cl}
1 & \text { für } \xi \in[a, x], \\
n(x-\xi)+1 & \text { für } \xi \in[x, x+1 / n], \\
0 & \text { für } \xi>x+1 / n .
\end{array}\right.
$$

Sie sind stetig und konvergieren fallend gegen $\varphi(. ; x)$. Die $\Lambda\left(f_{n}(. ; x)\right)$ bilden eine fallende Folge positiver Elemente aus $\Re . \quad \Phi(x)$ ist definitionsgemäss ihre untere Grenze ${ }^{1}$ ) (vgl. Nr. 1, Bedingung 2). Wegen $f_{n}(. ; x)<f_{n}\left(. ; x^{\prime}\right)$ und entsprechend $\Lambda\left(f_{n}(. ; x)\right) \leqq \Lambda\left(f_{n}\left(. ; x^{\prime}\right)\right)$ für $x<x^{\prime}$ definiert $x \rightarrow \Phi(x), \quad x \in[a, b]$, eine wachsende Abbildung von $[a, b]$ in $\Re$; es ist $\Phi(b)=\Lambda(1)$.

2) Beweis der Gleichung (1). Für Treppenfunktionen der Form

$$
t=\sum_{\nu} c_{\nu} \varphi\left(. ; x_{\nu}\right), \quad a<x_{1}<\ldots<x_{n}=b, \quad c_{\nu} \text { reell }
$$

setzen wir

$$
J(t)=\sum_{v} c_{v} \Phi\left(x_{v}\right)
$$

und zeigen: Ist $f \in C[a, b]$ und $f<t$, so gilt $\Lambda(f) \leqq J(t)$. Aus der Linearität von $A$ und $J$ folgt dann auch: Ist $f \in C[a, b]$ und $t<f$, so gilt $J(t) \leqq \Lambda(f)$.

Zum Beweis zerlegen wir die Summe (3) in zwei Teilsummen: die erste, $\Sigma^{\prime}$, wird über jene Glieder erstreckt, wo $c_{\nu}>0$ ist, die zweite, $\Sigma^{\prime \prime}$, dann über jene, wo $c_{v}<0$ ist. Wir setzen

$$
t^{\prime}=\sum_{v}^{\prime} c_{v} \varphi\left(. ; x_{v}\right), \quad t^{\prime \prime}=\sum_{v}^{\prime \prime} c_{v} \varphi\left(. ; x_{v}\right) .
$$

Dann ist $t=t^{\prime}+t^{\prime \prime}$, und aus $f<t$ folgt $-t^{\prime \prime}<t^{\prime}-f$ oder

und somit

$$
\sum_{\nu}^{\prime \prime}\left(-c_{\nu}\right) \varphi\left(. ; x_{\nu}\right)<\sum_{\nu}^{\prime} c_{\nu} \varphi\left(. ; x_{\nu}\right)-f
$$

$$
\sum_{\nu}^{\prime \prime}\left(-c_{\nu}\right) \varphi\left(. ; x_{\nu}\right)<\sum_{\nu}^{\prime} c_{\nu} f_{n}\left(. ; x_{\nu}\right)-f=g
$$

für jedes $n$. Rechts steht eine stetige Funktion $g$. Für ein genügend grosses $n$ gilt deshalb

$$
\sum_{v}^{\prime \prime}\left(-c_{v}\right) f_{n}\left(. ; x_{\nu}\right)<g .
$$

Da $\Lambda$ positiv ist, folgt

1) Diese ist unabhängig von der speziellen Wahl der $f_{n}$. 


$$
\sum_{\nu}^{\prime \prime}\left(-c_{v}\right) \Lambda\left(f_{n}\left(. ; x_{v}\right)\right) \leqq \Lambda(g)
$$

Dies ergibt

$$
\sum_{\nu}^{\prime \prime}\left(-c_{\nu}\right) \Phi\left(x_{\nu}\right) \leqq \sum_{\nu}^{\prime} c_{\nu} \Lambda\left(f_{n}\left(\cdot ; x_{\nu}\right)\right)-\Lambda(f)
$$

für alle $n$. Aus der Bemerkung in Nr. 1 folgt

$$
\sum_{\nu}^{\prime \prime}\left(-c_{v}\right) \Phi\left(x_{v}\right) \leqq \sum_{\nu}^{\prime} c_{\nu} \Phi\left(x_{v}\right)-\Lambda(f)
$$

oder

$$
\Lambda(f) \leqq J(t)
$$

Es sei nun $\varepsilon>0$. Es gibt eine Treppenfunktion $t$, sodass $t<f<t+\varepsilon$. Dann gilt nach dem soeben Gesagten

$$
J(t) \leqq \Lambda(f) \leqq J(t+\varepsilon)=J(t)+\varepsilon \Lambda(1)
$$

und nach Bemerkung 1 in Nr. 2

$$
J(t) \leqq \int_{a}^{b} f(x) d \Phi(x) \leqq J(t)+\varepsilon \Lambda(1) .
$$

Dies ergibt

$$
-\varepsilon \Lambda(1) \leqq \Lambda(f)-\int_{a}^{b} f(x) d \Phi(x) \leqq \varepsilon \Lambda(1),
$$

und da $\varepsilon \quad(>0)$ beliebig war, die Gleichung (1).

Wie man bemerkt haben wird, wurde in diesem Beweis die Bedingung $4 \mathrm{~b}$ in Nr. 1 nicht benützt.

4. Verallgemeinerung der Poisson-Stieltjes'schen Integraldarstellung. Es sei $U$ eine im Einheitskreis $\{z|| z \mid<1\}$ definierte Funktion mit Werten in $\Re$. Sie heisst harmonisch, wenn sie als Funktion von $x$ und $y \quad(z=x+i y)$ zweimal stetig differenzierbar ist und der Laplace'schen Gleichung $\Delta U=0$ genügt. Völlig analog zum Fall des reellen $U$ zeigt man, dass die im Einheitskreis harmonischen Funktionen $U$ (mit Werten in $\Re$ ) eine Entwicklung der Form

$$
U\left(r e^{i \varphi}\right)=\sum_{n=-\infty}^{\infty} C_{n} r^{|n|} e^{i n \varphi}
$$

$C_{0} \in \Re, \quad C_{p}=A_{p}+i B_{p}, \quad C_{-p}=A_{p}-i B_{p}, \quad A_{p}, B_{p} \in \Re, \quad p=1,2, \ldots$, besitzt. Sie konvergiert für $0 \leqq r<1$ absolut, d. h. 


$$
\sum_{n=1}^{\infty}\left\|A_{n}\right\| r^{n} \quad \text { und } \quad \sum_{n=1}^{\infty}\left\|B_{n}\right\| r^{n}
$$

konvergieren, und ist daher für jedes feste $r<1$ in $\varphi$ gleichmässig konvergent. Es gilt

Satz 1. Ist $U$ eine im Einheitskreise harmonische und positive Funktion mit Werten in $\Re$, so gibt es eine wachsende Abbildung $\Phi$ des Intervalls $[0,2 \pi]$ in $\Re$, sodass

$$
U(z)=\int_{0}^{2 \pi} \frac{1-|z|^{2}}{\left|e^{i \vartheta}-z\right|^{2}} d \Phi(\vartheta), \quad|z|<1 .
$$

Beweis. $\quad C_{2 \pi}$ bezeichne den Raum der komplexwertigen stetigen Funktionen von $e^{i \varphi}$ mit der Norm $\|f\|=\max _{\varphi}\left|f\left(e^{i \varphi}\right)\right| \cdot \quad U$ besitzt die Entwicklung (4). Da $U \geqq 0$ ist, gibt es eine positive lineare Abbildung $\Lambda$ von $C_{2 \pi}$ in $\Re$ mit $A\left(e^{-i n \varphi}\right)=C_{n}$. Ist nämlich $P$ die Klasse der komplexwertigen trigonometrischen Polynome $p$ :

und setzen wir

$$
p\left(e^{i \varphi}\right)=\sum_{k=-n}^{n} a_{k} e^{-i k \varphi}
$$

$$
\Lambda(p)=\sum_{k=-n}^{n} a_{k} C_{k}
$$

so ist $\Lambda$ auf $P$ linear und positiv. Denn für $p \geqq 0$ ist

$$
2 \pi \sum_{k=-n}^{n} a_{k} C_{k} r^{|k|}=\int_{0}^{2 \pi} U\left(r e^{i \varphi}\right) p\left(e^{i \varphi}\right) d \varphi \geqq 0
$$

und der Grenzübergang $r \rightarrow 1$ ergibt unter Benützung von Bedingung 4b in Nr. $1 \quad \Lambda(p) \geqq 0$. Gemäss (2) ist $A$ auf $P$ beschränkt und lässt sich deshalb unter Benützung derselben Bedingung zu einer positiven (und daher beschränkten) linearen Abbildung von $C_{2, \tau}$ in $\Re$ erweitern, wie oben behauptet wurde.

$z=r e^{i \varphi}$ gesetzt, hat der Poisson'sche Kern die Entwicklung

$$
P(\vartheta, z)=\frac{1-|z|^{2}}{\left|e^{i \vartheta}-z\right|^{2}}=\sum_{n=-\infty}^{\infty} r^{|n|} e^{i n(\varphi-\vartheta)} .
$$

Als Funktion von $\vartheta$ gehört er für $r<1$ zur Klasse $C_{2 \pi}$, und wegen der in $\vartheta$ gleichmässigen Konvergenz und der Beschränktheit von $A$ ist 


$$
\Lambda_{\vartheta}(P(\vartheta, z))=\sum_{n=-\infty}^{\infty} C_{n} r^{|n|} e^{i n \varphi}=U\left(r e^{i \varphi}\right) .
$$

Nach dem Lemma in Nr. 2 existiert ein $\Phi$ mit

$$
\Lambda_{\vartheta}(P(\vartheta, z))=\int_{0}^{2 \pi} P(\vartheta, z) d \Phi(\vartheta)
$$

was zusammen mit der vorangehenden Gleichung die Behauptung des Satzes gibt.

5. Analytische Funktionen mit positivem Realteil. Es sei $\mathfrak{c}$ ein komplexer Banach'scher Raum mit einer Involution *, d. i. $(A+B)^{*}$ $=A^{*}+B^{*}, \quad(c A)^{*}=\bar{c} A^{*}, \quad\left\|A^{*}\right\|=\|A\|$ und $A^{* *}=A$. Die Fixpunkte dieser Involution sind die sogenannte reellen Elemente in $\mathfrak{C}$; sie bilden einen reellen Banach'schen Raum, den reellen Unterraum in $\mathfrak{C}$. Jedes $Z \in \mathfrak{C}$ ist eindeutig in der Form $Z=X+i Y, X$ und $Y$ wreell», darstellbar; $\quad X=\left(Z+Z^{*}\right) / 2$ ist der Realteil, $\quad Y=\left(Z-i Z^{*}\right) /(2 i)$ der Imaginärteil von $Z$.

Voraussetzung. (5 sei ein komplexer Banach'scher Raum mit einer Involution *. Der diesbezügliche reelle Unterraum sei ein Raum $\Re$ im Sinne der Nr. 1.

Die im Einheitskreis definierte Funktion $F$ mit Werten in $\subseteq$ sei dort analytisch, d. h. in eine für $|z|<1$ konvergente Potenzreihe

$$
\sum_{n=0}^{\infty} C_{n} z^{n}
$$

entwickelbar. Dann hat der Realteil

$$
U\left(r e^{i \varphi}\right)=\frac{1}{2}\left(F\left(r e^{i \varphi}\right)+F\left(r e^{i \varphi}\right)^{*}\right)
$$

die Entwicklung

$$
U\left(r e^{i \varphi}\right)=\sum_{n=-\infty}^{\infty} A_{n} r^{\mid n} e^{i n \varphi},
$$

$A_{0}=\frac{1}{2}\left(C_{0}+C_{0}^{*}\right), A_{n}=C_{n}$ für $n=1,2, \ldots, A_{n}=C_{-n}^{*}$ für $n=-1,-2, \ldots$

Es gilt

Satz 1'. Es sei $F$ eine analytische Funktion im Einheitskreis mit Werten in 5 und mit positivem Realteil. Dann gibt es eine wachsende Abbildung $\Phi$ von $[0,2 \pi]$ in den reellen Unterraum von $\mathfrak{C}$, sodass

$$
F(z)=\int_{0}^{2 \pi} \frac{e^{i \vartheta}+z}{e^{i \vartheta}-z} d \Phi(\vartheta)+i \operatorname{Im} F(0), \quad|z|<1 .
$$


6. Kontraktive Operatoren. Es sei $\mathfrak{A}$ die Banach'sche Algebra der beschränkten Operatoren auf einem komplexen Hilbertraum $\mathfrak{H}$. Bei Beschränkung auf die lineare Struktur ist $\mathfrak{A}$ ein Raum $\mathfrak{E}$ im Sinne von Nr. 5 .

$A \in \mathfrak{A} \quad$ sei ein kontraktiver Operator, d. h. $\quad\|A x\| \leqq\|x\|, \quad x \in \mathfrak{S}$, oder $\|A\| \leqq 1$. Das Spektrum von $A$ liegt in der abgeschlossenen Kreisscheibe $\{\lambda|| \lambda \mid \leqq 1\}$, und die Resolvente $R(\lambda)=(\lambda E-A)^{-1}$ besitzt die für $|\lambda|>1$ konvergente Entwicklung

$$
R(\lambda)=\sum_{n=0}^{\infty} \frac{A^{n}}{\lambda^{n+1}},
$$

ist also eine analytische Funktion mit Werten in $\mathfrak{A}$. Die Funktion

$$
F(\lambda)=\lambda^{-1}(E+\lambda A) R(1 / \lambda), \quad|\lambda|<1,
$$

hat positiven Realteil. Setzt man nämlich $R(1 / \lambda) x=\lambda y, \quad x \in \mathfrak{S}$, so wird

$$
\begin{gathered}
(F(\lambda) x, x)=((E+\lambda A) y,(E-\lambda A) y) \\
=\|y\|^{2}-|\lambda|^{2}\|A y\|^{2}+\lambda(A y, y)-\bar{\lambda}(y, A y),
\end{gathered}
$$

und wegen $\|A\| \leqq 1$ folgt dann

$$
\operatorname{Re}(F(\lambda) x, x) \geqq\left.|| y\right|^{2}\left(1-|\lambda|^{2}\right)
$$

oder

$$
\left(\left(F(\lambda)+F(\lambda)^{*}\right) x, x\right) \geqq 0 \quad \text { d. h. } \quad \operatorname{Re} F(\lambda) \geqq 0, \quad \mid \lambda_{\mid}<1 .
$$

Nach Satz 1' gibt es eine wachsende Abbildung $\Phi$ von $[0,2 \pi]$ in die Hermite'schen Operatoren, sodass (es ist $F(0)=E$ )

$$
F(\lambda)=\int_{0}^{2 \pi} \frac{e^{i \vartheta}+\lambda}{e^{i \vartheta}-\lambda} d \Lambda(\vartheta) .
$$

Durch Entwicklung des Kernes auf der rechten Seite und der Funktion $F$ auf der linken Seite nach Potenzen von $\lambda$ :

$$
\frac{e^{i \vartheta}+\lambda}{e^{i \vartheta}-\lambda}=1+2 \sum_{n=1}^{\infty} e^{-i n \vartheta} \lambda^{n}, \quad F(\lambda)=E+2 \sum_{n=1}^{\infty} A^{n} \lambda^{n} .
$$

folgt

Satz 2. Es sei A ein kontraktiver Operator auf einem Hilbertraum. Dann gibt es eine wachsende Abbildung $\Phi$ von $[0,2 \pi]$ in den Raum der Hermite'schen Operatoren, sodass 
(7)

$$
A^{n}=\int_{0}^{2 \pi} e^{-i n \vartheta} d \Phi(\vartheta), \quad n=0,1,2, \ldots
$$

Für $n=0$ ergibt (7)

$$
E=\int_{0}^{2 \pi} d \Phi(\vartheta) \quad \text { oder } \quad \Phi(2 \pi)=E:
$$

$\Phi$ ist eine verallgemeinerte Zerlegung der Einheit (vgl. [2]).

Bemerkung. Ist $A$ kontraktiv und $x \in \mathfrak{H}$, so ist

$$
f(\lambda)=\left((E+\lambda A)(E-\lambda A)^{-1} x, x\right) \quad \text { für } \quad|\lambda|<1
$$

eine komplexwertige holomorphe Funktion mit positivem Realteil. Daraus folgt dann in bekannter Weise die Existenz eines $\Phi$ mit

$$
\left(A^{n} x, y\right)=\int_{0}^{2 \pi} e^{-i n \vartheta} d(\Phi(\vartheta) x, y) .
$$

Dies ist eine Art schwache Form von (7) und kann direkt aus (7) gewonnen werden.

7. Anwendungen. 1. Für beliebige kontraktive Operatoren $A$ gilt

$$
A^{* n}=\int_{0}^{2 \pi} e^{i n \vartheta} d \Phi(\vartheta), \quad n=1,2, \ldots
$$

Ist $A$ unitär, d. h. $A^{*}=A^{-1}$, so wird durch (7) und (8) dem trigonometrischen Polynom

der Operator

$$
p\left(e^{i \vartheta}\right)=\sum_{k=-n}^{n} a_{k} e^{i k \vartheta}
$$

zugeordnet:

$$
\sum_{k=-n}^{n} a_{k} A^{k}
$$

$$
\int_{0}^{2 \pi} p\left(e^{i \vartheta}\right) d \Phi(\vartheta)=p(A) .
$$

Die Abbildung $p \rightarrow \Lambda(p), p \in P$, (vgl. Nr. 4) ist also auch multiplikativ: $\Lambda\left(p_{1} p_{2}\right)=\Lambda\left(p_{1}\right) \Lambda\left(p_{2}\right)$, und $\Lambda$ ist ein Ring-Homomorphismus von $C_{2 \pi}$ in $\mathfrak{C}$. Daraus folgt dann $\Phi(\vartheta) \Phi\left(\vartheta^{\prime}\right)=\Phi(\vartheta)$ für $\vartheta \leqq \vartheta^{\prime}$, d. h. $\Phi(\vartheta)$ ist ein Projektionsoperator und $\Phi$ ist eine (orthogonale) Zer- 
legung der Einheit: wir haben die Spektraldarstellung für unitäre Operatoren gewonnen. Diese ist charakteristisch für unitäre Operatoren; die Formel $n=1$ in (7) mit einer verallgemeinerten Zerlegung der Einheit liefert aber im allgemeinen keinen kontraktiven Operator. Dagegen ist der folgende Satz richtig.

Satz 3. Ist $\Phi$ eine wachsende Abbildung des Intervalls $[0,2 \pi]$ in die Hermite'schen Operatoren eines Hilbertraumes $\mathfrak{S}$ und sind für einen Operator $A$ auf $\mathfrak{H}$ alle Gleichungen (F) mit $n=0,1,2, \ldots$ und $A^{0}=E$ erfüllt, so ist $A$ eine Kontraktion.

Beweis. Gemäss (2) ist $\|A\| \leqq 2$, also die Reihe

$$
\sum_{n=0}^{\infty} A^{n} \lambda^{n}
$$

für $|\lambda|<1 / 2$ konvergent. Durch Entwicklung des Integranden in (6) folgt daher die Gleichung

$$
F(\lambda)=(E+\lambda A)(E-\lambda A)^{-1}=\int_{0}^{2 \pi} \frac{e^{i \vartheta}+\lambda}{e^{i \vartheta}-\lambda} d \Phi(\vartheta)
$$

zunächst für $|\lambda|<1 / 2$. Da der Integrand auf der rechten Seite im Einheitskreis holomorph ist, folgt dasselbe für $F$. Da $\Phi$ wachsend ist, hat $F$ positiven Realteil, d. h. es ist

$$
\left(\left(F(\lambda)+F(\lambda)^{*}\right) x, x\right)=2 \operatorname{Re}(F(\lambda) x, x)
$$

positiv für $x \in \mathfrak{S}$ und $|\lambda|<1$. Mit $y=(E-\lambda A)^{-1} x$ ist aber gemäss $\mathrm{Nr} .6$

$$
\operatorname{Re}(F(\lambda) x, x)=\|\left. y\right|^{2}\left(1-|\lambda|^{2} \|\left. A\right|^{2}\right),
$$

und dies muss für $|\lambda|<1$ positiv sein. Also ist $\| A \leqq 1$.

2. Nach einem Satz von M. A. Neumark ([2]) ist jede verallgemeinerte Zerlegung der Einheit im Hilbertraum $\mathfrak{S}$ die Projektion einer (orthogonalen) Zerlegung der Einheit in einem erweiterten Hilbertraum $\mathfrak{S}^{*}$ $\left(\mathfrak{H}_{\mathcal{C}} \subset \mathfrak{h}^{*}\right)$ : es gibt zu einer wachsenden Abbildung $\Phi$ von $[0,2 \pi]$ in die Hermite'schen Operatoren auf $\mathfrak{\mathfrak { H }}$ eine (orthogonale) Spektralschar $S(\vartheta), \quad 0 \leqq \vartheta \leqq 2 \pi, \quad$ in $\mathfrak{S}^{*}$ und eine Orthogonalprojektion $\Pi$ von $\mathfrak{H}^{*}$ auf $\mathfrak{\mathfrak { H }}$ mit $\Phi(\vartheta)=\Pi S(\vartheta)$. Aus $(7)$ folgt dann

$$
A=\int_{0}^{2 \pi} e^{-i \vartheta} d(\Pi S(\vartheta))=\Pi \int_{0}^{2 \pi} e^{-i \vartheta} d S(\vartheta)=\Pi U .
$$

$U$ ist unitär in $\mathfrak{S}^{*}$; also wird jeder kontraktive Operator auf $\mathfrak{H}$ durch Orthogonalprojektion aus einem unitären Operator in einem erweiterten Hilbertraum gewonnen. 
3. Für einen kontraktiven Operator $A$ ist der folgende Mittelwertsatz bekannt (vgl. [5]): F'ür jedes Element $x$ des Hilbertraumes konvergieren die arithmetischen Mittel

$$
\frac{A^{m} x+A^{m+1} x+\ldots+A^{m+n} x}{n+1}
$$

für $n \rightarrow \infty$ stark gegen ein Element $x^{*}$, das gegenüber $A$ invariant ist. Die Darstellung (7) liefert einen neuen Beweis für diesen Satz. $S$ sei der Sprung, den die Funktion $\Phi$ im Nullpunkt erleidet, wenn sie über das Intervall $[0,2 \pi]$ hinaus gemäss der Funktionalgleichung $\Phi(\vartheta+2 \pi)=\Phi(\vartheta)+E$ fortgesetzt wird:

Da die Folge

$$
S=\inf _{0<\vartheta} \Phi(\vartheta)+E-\sup _{\vartheta<2 \tau} \Phi(\vartheta) .
$$

$$
\left(\Phi\left(\frac{1}{n}\right)-\Phi\left(2 \pi-\frac{1}{n}\right)\right)_{1}^{\infty}
$$

fallend ist, gilt für jedes $x \in \mathfrak{H}$ im starken Sinne

$$
\lim _{n \rightarrow \infty}\left(\Phi\left(\frac{1}{n}\right)+E-\Phi\left(2 \pi-\frac{1}{n}\right)\right) x=S x .
$$

Dies ergibt zusammen mit

$$
\frac{A^{m}+\ldots+A^{m+n}}{n+1}=\int_{0}^{2 \pi}\left(\frac{1}{n+1} \sum_{k=m}^{m+n} e^{i k \vartheta}\right) d \Phi(\vartheta)
$$

nach einigen trivialen Abschätzungen für beliebige Folgen der $m$ und für alle $x \in \mathfrak{S}$

$$
\lim _{n \rightarrow \infty} \frac{A^{m}+\ldots+A^{m+n}}{n+1} x=S x
$$

Daraus folgt $A S=S$.

4. Es sei jetzt $S$ ein symmetrischer Operator auf $\mathfrak{H}$ mit dem Defektindex 0 für die obere Halbebene. Dann ist $R(\lambda)=(A-\lambda E)^{-1}$ in der oberen Halbebene $\operatorname{Im} \lambda>0$ holomorph. Setzen wir $(A-\lambda E) y$ $=x$, so ist

$$
(R(\lambda) x, x)=(y,(A-\lambda E) y)=-\bar{\lambda}\|y\|^{2}+(y, A y) .
$$

Daraus folgt

$$
\left(\left(R(\lambda)-R(\lambda)^{*}\right) x, x\right)=(\lambda-\bar{\lambda})\|y\|^{2},
$$


d.h. $R(\lambda)$ hat positiven Imaginärteil. Durch konforme Verpflanzung auf den Einheitskreis (mit $\infty \rightarrow 1$ ) ergibt sich eine Darstellung der Form (6), und mit Methoden, die zu den in [4] verwendeten ganz analog sind, folgt dann die Existenz einer wachsenden Abbildung $\Psi$ von $(-\infty, \infty)$ in die Hermite'schen Operatoren auf $\mathfrak{H}$ mit

$$
S=\int_{-\infty}^{\infty} t d \Psi(t) .
$$

Eine etwas schwächere Form ist von M. A. Neumark ([2] und [3]) durch Erweiterung des gegebenen Hilbertraumes erhalten worden.

\section{Nachtrag (28. August 1963)}

Nach Schluss der Redaktion wurde mir bekannt, dass die in Nr. 7.2 genannte Eigenschaft der kontraktiven Operatoren, Projektionen von unitären Operatoren in einem Erweiterungsraum zu sein, schon von P. R. Halmos [1] bewiesen wurde. Für Potenzen kontraktiver Operatoren vergleich auch B. Sz.-Nagy [6]-[7].

Eidgenössische Technische Hochschule

Zürich, Schweiz

\section{Literatur}

[1] Halmos, P. R.: Normal dilations and extensions of operators. - Summa Brasil. Math. 2, 1950, S. 125-134.

[2] НАймарк, М. А.: О самосопряженных расширениях второго рода спмметрического оператора. - Изв. Акад. Наук СССР. Сер. Мат. 4, 1940, \$. 53-104.

[3] -»- Спектральные функции симметрического оператора. - ІІзв. Акағ. Наук СССР. Cep. Мат. 4, 1940, S. $277-318$.

[4] Nevanlinna, F., und T. Nieminen: Das Poisson-Stieltjes'sche Integral und seine Anwendung in der Spektraltheorie des Hilbert'schen Raumes. - Commentationes in Honorem Rolf Herman Nevanlinna die natali eius sexagesimo. Ann. Acad. Scient. Fennicæ A. I. 207, 1955.

[5] Riesz, F., und B. v. Sz. NAGY: Über Kontraktionen des Hilbertschen Raumes. Acta Sci. Math. (Szeged) 10, 1943, S. 202-205.

[6] Sz.-Nagy, B.: Sur les contractions de l'espace de Hilbert. - Acta Sci. Math. (Szeged) 15, 1953/1954, S. 87-92.

[7] - $\gg$ - Transformations de l'espace de Hilbert, fonctions de type positif sur un groupe. - Acta Sci. Math. (Szeged) 15, 1953/1954, S. 104-114. 